Marquette University

e-Publications@Marquette

Chemistry Faculty Research and Publications

Chemistry, Department of

$1-1-2007$

Use of Factor Analysis in Multi-Electron Spectroelectrochemistry

Michael D. Ryan

Marquette University, michael.ryan@marquette.edu

Robert L. Keesey

Marquette University

Published version. Published as part of AIP Conference Proceedings, 2007. DOI. (C) 2007 American Institute of Physics. Used with permission. 


\title{
Use of Factor Analysis in Multi-Electron Spectroelectrochemistry
}

\author{
Michael D. Ryan and Robert L. Keesey \\ Marquette University, Chemistry Dept., PO Box 1881, Milwaukee, WI 53092 USA
}

\begin{abstract}
Spectroelectrochemistry and voltammetry contain both unique and complementary information. For multielectron transfers, information on each electron exchange is only directly accessible in the voltammetric data if the potentials are well separated so that two distinct waves can be observed. If the $\mathrm{E}^{\circ}$ 's are close together, the voltammetric data will contain the sum of the two exchanges which can only be deconvoluted by modeling the system and solving the appropriate equations. On the other hand, the spectroscopic data contains direct information on each electron exchange even when the $\mathrm{E}^{\circ}$ 's are close together. Unfortunately, this information cannot be readily extracted if the intermediate oxidation state does not have a potential region where it is the dominant species. Chemometric methods such as factor analysis though can be used to deduce the spectra of each species even if they don't dominate in any potential region. Initial work on the application of factor analysis to spectroelectrochemistry has been reported. Traditional methods of electroanalytical analysis are based on models that relate the concentration of electroactive materials to electrode potentials and solution concentrations. The model and parameters are adjusted to obtain the best fit to a model. Chemometric methods such as factor analysis allow the experimenter to determine solution concentrations without knowledge of the precise electrochemical mechanism. The utility of this approach will be demonstrated by the study of a protein, E. coli sulfite reductase hemoprotein, which is capable of transferring two-electrons and the $\Delta \mathrm{E}^{\circ}$ values are less than $100 \mathrm{mV}$, causing the waves to overlap. With these methods more detailed information on the electron transfer rate and associated kinetics processes can be more clearly identified.
\end{abstract}

Keywords: spectroelectrochemistry, factor analysis, voltammetry, sulfite reductase, E. coli, electron transfer kinetics PACS: $82.45 . \mathrm{Rr}$

\section{INTRODUCTION}

Sulfite reductase catalyzes the 6-electron reduction of bisulfite to sulfide [1]. In vitro, sulfite reductase is also capable of reducing nitrite to ammonia [2]. The enzyme contains two prosthetic groups: an iron-siroheme and a $4 \mathrm{Fe}-4 \mathrm{~S}$ cluster, each capable of a one-electron reduction. The SiR-HP ${ }^{0}$ enzyme can be photoreduced to SiR-HP ${ }^{2-}$ with 5'-deazaflavin and EDTA. The visible bands of the fully oxidized enzyme at 388, 547, 591 and $714 \mathrm{~nm}$ decrease, and new bands are observed at 397 and $608 \mathrm{~nm}$ upon reduction [3]. The redox potential of the first reduction was found to be $-340 \mathrm{mV}$ vs NHE, while the second reduction was found to be $-405 \mathrm{mV}[3,4]$.

$$
\begin{aligned}
& {\mathrm{SiR}-H P^{0}}^{-\mathrm{e}^{-}} \rightarrow \text { SiR-HP }^{1-} \\
& \text { SiR-HP }^{1-}+\mathrm{e}^{-} \rightarrow \text { SiR-HP }^{2-}
\end{aligned}
$$

where $\mathrm{SiR}-\mathrm{HP}^{0}$ is the fully oxidized enzyme, $\mathrm{SiR}-\mathrm{HP}^{1-}$ is the siroheme reduced enzyme (ferrosiroheme) $\left(\mathrm{E}_{0 / 1}^{\circ}\right)$, and SiR-HP ${ }^{2-}$ is the fully reduced enzyme (ferrosiroheme, $\left.[4 \mathrm{Fe}-4 \mathrm{~S}]^{+}\right)\left(\mathrm{E}_{1 / 2}^{\circ}\right)$. The SiR-HP enzyme can be directly reduced at the methyl viologen modified gold minigrid electrode $[5,6]$. The electron transfer can be monitored spectroscopically using visible spectroscopy, and the reduced enzyme can be completely recovered when the applied potential was sufficiently positive.

The kinetic parameters for the electron transfer and associated kinetic processes can be obtained from the concurrent acquisition of voltammetric and spectroscopic data during the linear potential scan. The $\mathrm{E}^{\circ} \mathrm{s}$ for the individual electron transfers are too close together for the one-electron reduced intermediate, $\mathrm{SiR}^{-} \mathrm{HP}^{1-}$, to be observed without the concomitant presence of either the fully reduced or fully oxidized enzyme. In order to

CP963, Vol. 2 Part A, Computation in Modern Science and Engineering, Proceedings of the International Conference on Computational Methods in Science and Engineering 2007, edited by T. E. Simos and G. Maroulis (C) 2007 American Institute of Physics 978-0-7354-0478-6/07/\$23.00 


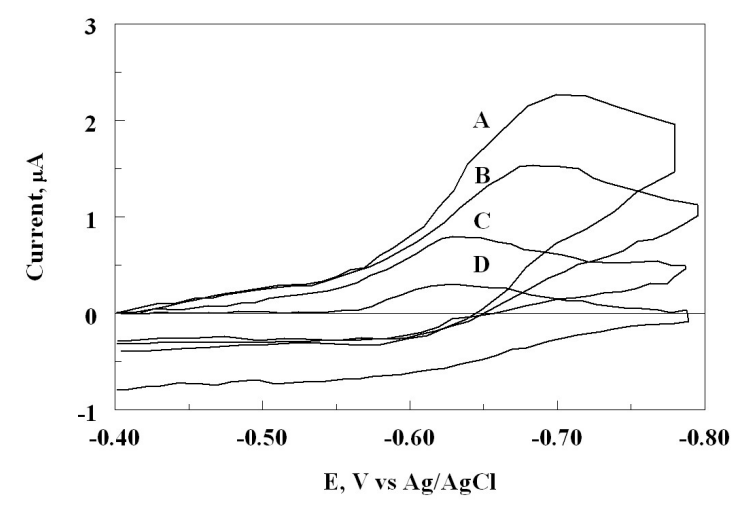

Figure $1 b$

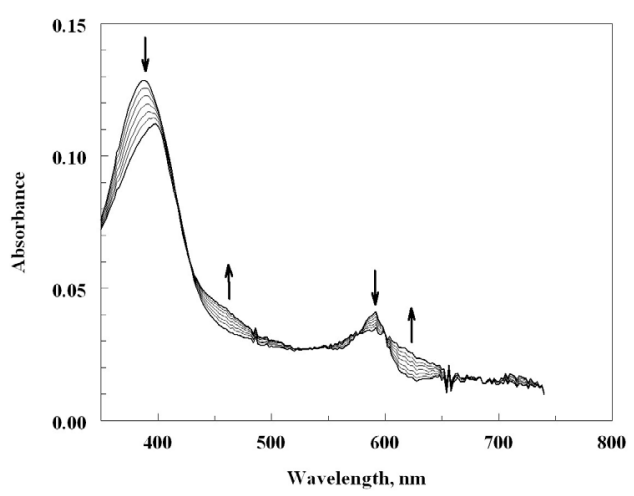

Figure 1a

FIGURE 1. a. Cyclic voltammetry of $0.71 \mathrm{mM} \mathrm{SiR-HP}{ }^{0}$. Scan rates: A. $1.5 \mathrm{mV} / \mathrm{s}$. B. $0.70 \mathrm{mV} / \mathrm{s}$. C. $0.30 \mathrm{mV} / \mathrm{s}$. D. $0.062 \mathrm{mV} / \mathrm{s}$. b. Spectroelectrochemistry of $0.71 \mathrm{mM} \mathrm{SiR}-\mathrm{HP}^{0}$. Scan rate: $1.5 \mathrm{mV} / \mathrm{s}$. Starting potential: $-0.40 \mathrm{~V}$, intermediate potentials: $-0.63 \mathrm{~V},-0.66 \mathrm{~V},-0.69 \mathrm{~V},-0.72 \mathrm{~V},-0.75 \mathrm{~V}$. Final potential : $-0.80 \mathrm{~V}$. All potentials versus $\mathrm{Ag} / \mathrm{AgCl}$. Temperature: $10^{\circ} \mathrm{C}$. $0.10 \underline{\mathrm{M}}$ phosphate buffer, $\mathrm{pH} 7.7$.

determine the concentration of each species at every potential, the spectra were deconvoluted using evolving factor analysis (EFA). EFA is a model-free deconvolution method, and is ideally suited for spectroelectrochemistry because each species appears sequentially in time. The basis of the technique is that the number of factors at each potential region can be determined from the number of eigenvalues that are present in that region which are significantly above the noise level. Initially in the spectrum, only one species (factor) is present ( $\mathrm{SiR}-\mathrm{HP}^{0}$ in this case). As the potential is scanned to more negative potentials, additional factors (SiR-HP ${ }^{1-}$ and $\mathrm{SiR}-\mathrm{HP}^{2-}$ ) will be observed in the analysis. EFA is then used to convert these factors to spectra and concentrations. From the concentrations, the flux (which is proportional to the current) can be calculated, and the mechanism and parameters for the electron transfer processes can be more easily deduced.

\section{RESULTS AND DISCUSSION}

E. coli sulfite reductase hemoprotein $\left(\mathrm{SiR}-\mathrm{HP}^{0}\right)$ can be directly reduced at a methyl viologen-modified gold electrode. The spectral were obtained with a diode array spectrophotometer with an acquisition time of $2 \mathrm{~s}$. A thinlayer cell with a thickness of $100 \mu$ was used so that there were little to no concentration polarization during the scan. The reduction can be observed either voltammetrically or spectroscopically. A typical set of voltammograms is shown in Fig.1a and spectroscopy data in Fig. 1b. Only a single wave was observed because the redox potentials are close together. The wave appears to be electrochemically irreversible because the oxidation is slower than the reduction. Spectroscopic data show that, while electrochemically irreversible, the process is chemically reversible. With the use of evolving factor analysis [5,7], the visible spectra of the three oxidation states were determined [5], and the concentration as a function of potential was determined (Fig. 2). The reductions did not occur at the thermodynamic potentials, indicating sluggish electron rates. On the positive scan, reduction of $\mathrm{SiR}^{-\mathrm{HP}^{0}}$ and $\mathrm{SiR}$ $\mathrm{HP}^{1-}$ continued until the potential approaches the $\mathrm{E}^{\circ}{ }_{1 / 2}$. Even by the end of the scan, very little SiR-HP ${ }^{1-}$ was oxidized back to SiR-HP ${ }^{0}$, though complete conversion occurred at slower scan rates. This behavior is indicative of a slow kinetics process (e.g., conformational changes/ligand exchange) which must occur prior to the electron transfer. This slow ferric/ferrous siroheme electron transfer is consistent with other kinetic studies of the enzyme.

Analysis of an EE mechanism directly from the voltammetry is complicated by the background current, especially when metal electrodes are used. The background current will include both surface reactions and charging current, making it difficult to separate the faradaic from non-faradaic currents. This technique, derivative cyclic voltabsorptometry (DCVA), has been applied to one-electron transfer processes. The morphological equivalence of DCVA and cyclic voltammograms has already been pointed out for single electron transfers [8]. Its application to the EE mechanism, though, would be much more advantageous in that the current for each electron transfer could be 

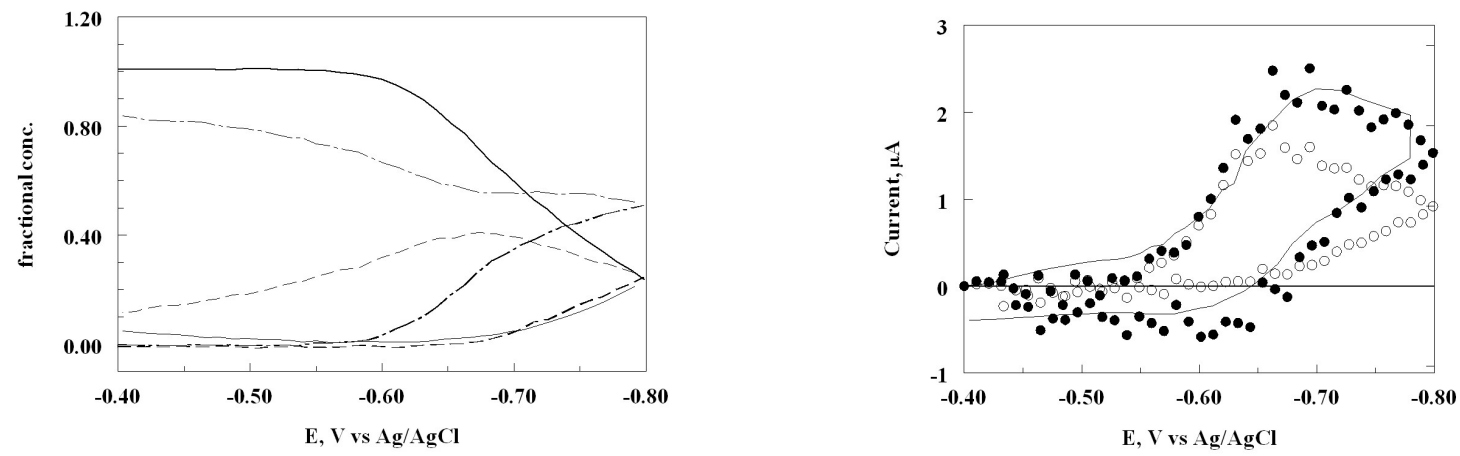

FIGURE 2. Fractional concentrations of $0.71 \mathrm{mM} \mathrm{SiR-HP}$ as a function of potential. Solid line: SiR-HP0, dash/dot line: SiRHP1-, Dash line: SiR-HP2-. Thick lines: forward scan; thin lines: reverse scan. Scan rate: $1.5 \mathrm{mV} / \mathrm{s}$. Temperature: $10^{\circ} \mathrm{C}$. $\mathrm{pH} 7.7$.

FIGURE 3. Comparison of cyclic voltammetric current from the DCVA current calculated from Fig. 3. Solid line: cyclic voltammetric data; Filled circles: total DCVA current; Open circle: $i_{1}$.

separated and analyzed individually. This is, of course, not possible from the cyclic voltammetric data, but can be done by transformation of the spectroscopic data

The current is proportionational to the flux of the electroactive species. For the first electron transfer in a thinlayer geometry, this is given by:

$$
i_{1}=-F V \frac{d\left[S i R-H P^{0}\right]}{d t}=-F V v \frac{d\left[S i R-H P^{0}\right]}{d E}
$$

Where $i_{1}=$ current due to the first electron transfer, $F=$ Faraday's constant, $v=$ scan rate $(\mathrm{V} / \mathrm{s}), E=$ potential $(\mathrm{V})$, and $V=$ volume of the solution. The current for the second electron transfer $\left(i_{2}\right)$ can be calculated similarly. The total current $\left(i_{\text {total }}\right)$ is equal to:

$$
i_{\text {total }}=F V v\left(\frac{d\left[S i R-H P^{2-}\right]}{d E}-\frac{d\left[S i R-H P^{0}\right]}{d E}\right)
$$

Using Eq. 3 and 4 above and the data in Fig. 2, the total normalized current was calculated from the spectroscopic data and is shown in Fig. 3, superimposed with the cyclic voltammetric data. Excellent agreement was obtained. On the same axes, the DCVA current calculated from the first electron transfer is shown. The reduction current was mostly due to the first electron transfer at this scan rate, but the oxidation current was due primarily to the oxidation of SiR-HP ${ }^{2-}$ that was formed in the forward scan. Very little current was due to the oxidation of SiR-HP ${ }^{1-}$ to SiR$\mathrm{HP}^{0}$.

These analyses were performed over a range of scan rates. The results clearly show that slow electron transfer alone does not account for the voltammetric behavior. In particular, there is considerable hysteresis in the first electron transfer process. The initial reduction corresponds well to a quasi-reversible electron transfer, but there appears to be a slow chemical step in the re-oxidation, probably involving ligand exchange at the iron siroheme site (water/anion exchange).

Spectroelectrochemistry and cyclic voltammetry of SiR-HP in the presence of cyanide was also performed. As was observed when a strong ligand was absent, the spectroscopic data could be deconvoluted by the use of factor analysis into three spectroscopic species. Previous evidence has shown that cyanide remained coordinated in all three oxidation states [4]. The electrochemical mechanism for the cyanide coordinated enzyme was more reflective of an EE mechanism with quasi-reversible electron transfer. At slow scan rates, the cyanide bound enzyme was consistent with a EE mechanism with slow electron transfer. By using DCVA and separating the two currents, the $\mathrm{E}^{\circ}$ values for the $1^{\text {st }}$ and $2^{\text {nd }}$ electron transfer were found to be $-426 \mathrm{mV}$ and $-496 \mathrm{mV}$ vs $\mathrm{Ag} / \mathrm{AgCl}$. The quasireversibility of the electron transfers without additional kinetic complications indicated that conformation 
change/ligand exchange was not necessary in the cyanide bound enzyme in contrast with the voltammetric/spectroscopic behavior observed in the absence of strong ligands.

\section{CONCLUSIONS}

The combination of chemometric, spectroscopic and electroanalytical methods has been shown to be quite powerful in the analysis of multi-electron transfers. The ability to experimentally ascribe the observed voltammetric current to each individual electron transfer gives the analysts additional insight into this important mechanism.

\section{REFERENCES}

1. Siegel, L. M., Davis, P. S., and Kamin, H., J. Biol. Chem. 249, 1572-1586 (1974).

2. Krueger, R. J. and Siegel, L. M., Biochemistry 21, 2892-2904 (1982).

3. Janick, P. A. and Siegel, L. M., Biochemistry 21, 3538-3547 (1982).

4. Siegel, L. M., Rueger, D. C., Barber, M. J., Krueger, R. J., Orme-Johnson, N. R., and Orme-Johnson, W. H., J. Biol. Chem. 257, 6343-6350 (1982).

5. Keesey, R. L. and Ryan, M. D., Anal. Chem. 71, 1744-1752 (1999).

6. M. D. Ryan and P. W. Crawford, Multi-electron transfer spectroelectrochemistry: application to E. coli sulfite reductase, Proceedings of the Fifth International Symposium on Redox Mechanisms and Interfacial Properties of Molecules of Biological Importance,(The Electrochemical Society, Princeton, NJ, 1993).

7. E. R. Malinowski, Factor Analysis in Chemistry,2 ed.(John Wiley \& Sons, Inc., New York, 1991).

8. Bancroft, E. E., Sidwell, J. S., and Blount, H. N., Anal. Chem. 53, 1390-1394 (1981). 